\title{
Introduction to the Minitrack on Wearable Technology and the Internet of Things
}

\author{
Jan Kietzmann \\ Simon Fraser University \\ jkietzma@sfu.ca
}

\author{
Leyland Pitt \\ Simon Fraser University \\ lpitt@sfu.ca
}

\author{
Ian McCarthy \\ Simon Fraser University \\ imccarth@sfu.ca
}

\author{
Hope Schau \\ University of Arizona \\ hschau@email.arizona.edu
}

The Internet of Things (IoT) refers to a computing concept that allows everyday physical objects to be connected to the Internet so that these are able to identify themselves to other devices and engage in seamless and automatic data exchange. The IoT is receiving more and more attention among researchers, especially as connecting "things" (e.g., jet engines, manufacturing equipment) steadily moves towards Gartner's Slope of Enlightenment.

At the same time as the digital and industrial worlds merge, and possibly collide through the IoT, the phenomenon of wearable technologies is quickly emerging, allowing users to monitor, control, optimize and even autonomize a wide range of functions and behaviors. As these new devices become equal actors alongside connected "things" in the real world and purely virtual entities online, they extend people's roles from being mere users and observers of the Internet, to becoming part of the Internet - what might indeed be termed the Internet of People (IoP).

The combination of IoT, wearables and the IoP leads us to the Internet of Everything (IoE). The opportunities and challenges that the new data stocks and flows of the IoE will create for organizations, governments, individuals and society are the focus of this minitrack.
The presentations will begin with Wei-Yen Hsu who will talk about the application of brain switch control to daily life. This will be followed by Mikael Wiberg's presentation on the design and development of a prototype system that integrates the Internet of Things (IoT) technologies, architecture and mobile interaction. After this, Mashood Ahmad, Marcel Papert, and Alexander Pflaum will talk about Dynamic capabilities related implementation skills for Internet of Things solutions in the digital economy. Finally, Liana Furini will present about machine learning with thoughts about the future of IoT.

After our four paper presentations, for the remainder of our minitrack session, we will lead an interactive discussion between the audience, our paper presenters and our panel of IoE experts. This panel will include Karen Robson (Professor of Marketing at Central Michigan University), David Hannah (Professor of Management and Organization Studies at Simon Fraser University), Leyland Pitt (Professor of Management and Organization Studies at Simon Fraser University), and Hope Schau (Professor of Marketing/ Entrepreneurship and Innovation at the University of Arizona). 